\title{
Delay-range-dependent Robust Stability for Uncertain Singular Systems with Interval Time-varying Delays
}

\author{
Pin-Lin Liu \\ Department of Automation Engineering Institute of Mechatronoptic System, Chienkuo Technology University, Taiwan \\ *Corresponding Author: E-mail:Ipl@cc.ctu.edu.tw
}

Copyright (C) 2013 Horizon Research Publishing All rights reserved.

\begin{abstract}
In this paper, we consider the delay-rangedependent robust stability problem of uncertain singular system with interval time-varying delays. Stability criteria, which guarantee the concerned singular system is regular, free and stable, is derived in terms of linear matrix inequalities (LMIs) by introducing some identical equations with integral inequality approach (IIA) and using the Leibniz-Newton formula to reduce the conservatism of the results. The upper bound of time-delay can be obtained by using the modified generalized eigenvalue minimization problem (GEVP) technique such that the system can be stabilized for all time-delays. Finally, numerical examples show the method presented in our paper is more effective and less conservative than the existing ones.
\end{abstract}

Keywords Singular Time Delay Systems, Integral Inequality Approach (IIA), Delay- Dependence, Linear Matrix Inequality (LMI)

\section{Introduction}

Engineering processes often involve both nonlinear and time-delay models. In many physical and biological phenomena, the rate of variation in the system state depends on past states. Time delay phenomena were first discovered in biological systems and were later found in many engineering systems, such as mechanical transmissions, fluid transmissions, metallurgical processes, and networked control systems. They are often a source of instability and poor control performance. Therefore, many efforts have been made for the stability problems for various delayed systems [1-2, 4-14, 16-31]. Moreover, because of unavoidable factors, such as modeling error, external perturbation and parameter fluctuation, the time delay systems certainly involve uncertainties such as perturbations and component variations, which will change the stability of time delay systems. And in recent years, the stability analysis issues for time delay systems in the presence of parameter uncertainties perturbations have stirred some initial research attention $[4,5,8,12,16,19,20$, 22-25, 31].

Singular systems have found numerous practical applications: e.g., engineering systems, social systems, economic systems, biological systems. Unlike classical state space representation via a set of ordinary differential equations, singular system can be viewed as a composite formed by several interconnected systems with two layers: dynamic property described by differential equation and interconnection property expressed by algebraic equation. Therefore, some results on the stability of singular systems are achieved [1, 5-8, 12-13, 16-18, 20-25, 27-30] and the references therein. The existing stability criteria for singular time-delay systems can be classified into two types: delayindependent $[5,24,25,30]$ and delay-dependent $[1,6-8$, $12-13,16-19,21-23,26,28-29,31]$. Generally, delaydependent conditions are less conservative than the delayindependent ones, especially when the time delay is small. For singular systems with delays, several kinds of simple Lyapunov-Krasovskii functionals, i.e. functionals parameterized with constant matrices, have been proposed, which lead to different levels of conservatism due to the different model transformations and the bounding techniques for some cross-terms [7, 8]. A tighter bounding for cross-terms can reduce the conservatism. However, there are no obvious ways to obtain less conservative results, even if one is willing to expend more computational effort on the problem, and to find a tighter bound for the cross-terms. This is the serious limitation for these criteria. To overcome this limitation, one has to find some more general Lyapunov-Krasovskii functional (LKF) for handling the delay-range-dependent robust stability problem for singular systems. To the best of our knowledge, this delay- range-dependent robust stability problem has not been fully investigated for singular systems with time-varying delay, which motivates the present study.

On the other hand, the range of time-varying delay systems considered in $[17,18,20,27]$ is from 0 to an upper bound. In practice, a time-varying interval delay is often encountered, that is, the range of delay varies in an interval for which the lower bound is not restricted to 0 . In this case, 
the stability criteria for time-varying delay systems in $[17,18$, $20,27]$ are conservative because they do not take into account the information of the lower bound of delay. To the best of authors' knowledge, there have been few results on the delay-range-dependent robust stability of the singular systems with time-varying interval delays, which remain as an interesting research topic.

Motivated by the above discussions, we propose the improved delay-range-dependent robust stability for singular systems with time-varying interval delay and uncertainties. First, by defining a novel Lyapunov function, a delay-range-dependent stability criterion for the nominal singular time- delay system is established in terms of LMIs. Next, less conservative result is obtained by considering some useful terms when estimating the upper bound of the derivative of Lyapunov functional and introducing the additional terms into the proposed Lyapunov functional, which includes the information of the range. The maximum allowable value of the time delay can be obtained by solving a set of linear matrix inequalities (LMIs) and the modified generalized eigenvalue minimization problem (GEVP) algorithm. Finally, Numerical examples demonstrate that the results obtained in this paper are effective and are a significant improvement over previous ones.

\section{Stability Description and Preliminaries}

Consider the following uncertain singular system with an interval time-varying delay as follows:

$$
E \dot{x}(t)=(A+\Delta A(t)) x(t)+(B+\Delta B(t)) x(t-h(t)), \quad \mathrm{t}>0,
$$

with the initial condition

$$
x(t)=\phi(t), t \in\left[-h_{2}, 0\right],
$$

where $x(t) \in R^{n}$ is the state vector of the system; $A, B \in R^{n \times n} \quad$ are constant matrices; The matrix $E \in R^{n \times n}$ maybe singular, without loss generality, we suppose $\operatorname{rankE}=r \leq n ; \phi(t)$ is a continuously real-valued initial function on $\left[-h_{2}, 0\right] . h(t)$ is a time-varying delay which satisfies:

$$
h_{1} \leq h(t) \leq h_{2}, \quad \dot{h}(t) \leq h_{d}<1, \quad \forall t \geq 0,
$$

where $h_{1}$ and $h_{2}$ are the lower and upper delay bounds, respectively, $h_{1}, h_{2}$ and $h_{d}$ are constants and $0 \leq h_{1}<h_{2}$. Time-varying parametric uncertainties $\Delta A(t)$ and $\Delta B(t)$ are assumed to be of the following form:

$$
[\Delta A(t) \quad \Delta B(t)]=M F(t)\left[\begin{array}{ll}
N_{a} & N_{b}
\end{array}\right],
$$

where $M, N_{a}$, and $N_{b}$ are known real constant matrices with appropriate dimensions, and $F(t)$ is an unknown, real, and possibly time-varying matrix with Lebesguemeasurable elements satisfying

$$
F^{T}(t) F(t) \leq I, \quad \forall t .
$$

The main objective is to find the range of $h_{1} \leq h(t) \leq h_{2}$ and guarantee stability for the uncertain singular time-varying delay systems (1). Here, definitions fundamental lemmas are reviewed.

Definition 1 [3]: The pair $(E, A)$ is said to be regular if $\operatorname{det}(s E-A)$ is not identically zero.

Definition 2[3]: The pair $(E, A)$ is said to be impulse free if $\operatorname{deg}(\operatorname{det}(s E-A))=\operatorname{rank} E$.

Definition 3[3]: For a given scalar $\bar{h}>0$, the singular time-varying delay system (1) is said to be regular and impulse free for any constant time delay $h$ satisfying $0 \leq h \leq \bar{h}$, it the pairs $(E, A)$ and $(E, A+B)$ are regular and impulse free.

Remark 1:The regularity and the absence of impulses of the pair $(E, A)$ ensures the system (1) with time delay $h \neq 0$ to be regular and impulse free, while the fact that the pair $(E, A+B)$ is regular and impulse free ensures the system (1) with time delay $h=0$ to be regular and impulse free.

Lemma 1 [15]: The singular system $E \dot{x}(t)=A x(t)$ is regular, impulse free, and stable, if and only if there exists a matrix $P$ such that:

$$
A^{T} P+P A<0,
$$

with the following constraint

$$
P^{T} E=E^{T} P \geq 0 .
$$

Lemma 2 [17]: For any positive semi-definite matrices:

$$
X=\left[\begin{array}{ccc}
X_{11} & X_{12} & X_{13} \\
X_{12}^{T} & X_{22} & X_{23} \\
X_{13}^{T} & X_{23}^{T} & X_{33}
\end{array}\right] \geq 0,
$$

the following integral inequality holds

$$
\begin{aligned}
& -\int_{t-h(t)}^{t} \dot{x}^{T}(s) X_{33} \dot{x}(s) d s \\
& \leq \int_{t-h(t)}^{t}\left[x^{T}(t) \quad x^{T}(t-h(t)) \quad \dot{x}^{T}(s)\right] \times \\
& {\left[\begin{array}{ccc}
X_{11} & X_{12} & X_{13} \\
X_{12}^{T} & X_{22} & X_{23} \\
X_{13}^{T} & X_{23}^{T} & 0
\end{array}\right]\left[\begin{array}{c}
x(t) \\
x(t-h(t)) \\
\dot{x}(s)
\end{array}\right] d s .}
\end{aligned}
$$

Lemma 3 [2]. The following matrix inequality:

$$
\left[\begin{array}{ll}
Q(x) & S(x) \\
S^{T}(x) & R(x)
\end{array}\right]<0,
$$

where $Q(x)=Q^{T}(x), R(x)=R^{T}(x)$ and $S(x)$ depend affine on $x$, is equivalent to

$$
\begin{aligned}
& R(x)<0, \\
& Q(x)<0,
\end{aligned}
$$


and

$$
Q(x)-S(x) R^{-1}(x) S^{T}(x)<0 .
$$

Lemma 4[2]. Given matrices $Q=Q^{T}, D, E$, and $R=R^{T}>0$ of appropriate dimensions,

$$
Q+D F(t) E+E^{T} F^{T}(t) D^{T}<0,
$$

for all $F(t)$ satisfying $F^{T}(t) F(t) \leq I$, if and only if there exists some $\varepsilon>0$ such that

$$
Q+\varepsilon D D^{T}+\varepsilon^{-1} E^{T} E<0 .
$$

In this paper, a new Lyapunov functional is constructed, which contains the information of the lower bound of delay $h_{1}$ and upper bound $h_{2}$.

The nominal unforced time delay singular system (1) can be written as follows:

$$
E \dot{x}(t)=A x(t)+B x(t-h(t)) .
$$

The following Theorem 1 presents a delay-range- dependent result in terms of LMIs and expresses the relationships between the terms of the Leibniz-Newton formula.

Theorem 1: For three given positive scalars $h_{1}, h_{2}$, and $h_{d}$, the time-delay singular system (9) is asymptotically stable if there exist matrices $P=P^{T}>0, Q_{i}=Q_{i}^{T}>0$, $R_{j}=R_{j}^{T}>0(i=1,2,3 ; j=1,2), \quad S \quad$ with appropriate dimensions, positive semi-definite matrices: $X=\left[\begin{array}{ccc}X_{11} & X_{12} & X_{13} \\ X_{12}^{T} & X_{22} & X_{23} \\ X_{13}^{T} & X_{23}^{T} & X_{33}\end{array}\right] \geq 0, \quad$ and $\quad Y=\left[\begin{array}{ccc}Y_{11} & Y_{12} & Y_{13} \\ Y_{12}^{T} & Y_{22} & Y_{23} \\ Y_{13}^{T} & Y_{23}^{T} & Y_{33}\end{array}\right] \geq 0$ such that the following LMIs hold:

$$
\begin{gathered}
\Xi=\left[\begin{array}{cccccc}
\Xi_{11} & \Xi_{12} & 0 & 0 & \Xi_{15} & \Xi_{16} \\
\Xi_{12}^{T} & \Xi_{22} & \Xi_{23} & \Xi_{24} & \Xi_{25} & \Xi_{26} \\
0 & \Xi_{23}^{T} & \Xi_{33} & 0 & 0 & 0 \\
0 & \Xi_{24}^{T} & 0 & \Xi_{44} & 0 & 0 \\
\Xi_{15}^{T} & \Xi_{25}^{T} & 0 & 0 & \Xi_{55} & 0 \\
\Xi_{16}^{T} & \Xi_{26}^{T} & 0 & 0 & 0 & \Xi_{66}
\end{array}\right]<0, \\
E^{T}\left(R_{1}-X_{33}\right) E \geq 0, \\
E^{T}\left(R_{2}-Y_{33}\right) E \geq 0,
\end{gathered}
$$

with the following constraint

$$
P^{T} E=E^{T} P \geq 0 .
$$

where $Z \in R^{n \times(n-r)}$ is any matrix with full column rank and satisfies $E^{T} Z=0$ and

$$
\begin{aligned}
\Xi_{11}= & A^{T} P+P A+A^{T} Z S^{T}+S Z^{T} A+Q_{1}+Q_{2}+Q_{3} \\
& +E^{T}\left(h_{2} X_{11}+X_{13}+X_{13}^{T}\right) E, \\
\Xi_{12}= & P B+S Z^{T} B+E^{T}\left(h_{2} X_{12}-X_{13}+X_{23}^{T}\right) E, \\
\Xi_{15} & =h_{2} A^{T} R_{1}, \Xi_{16}=h_{21} A^{T} R_{2},
\end{aligned}
$$

$$
\begin{aligned}
\Xi_{22} & =-\left(1-h_{d}\right) Q_{3}+E^{T}\left(h_{21} X_{11}+X_{13}+X_{13}^{T}+h_{2} X_{22}-X_{23}-X_{23}^{T}\right. \\
& \left.+h_{21} Y_{22}-Y_{23}-Y_{23}^{T}+h_{21} Y_{11}+Y_{13}+Y_{13}^{T}\right) E, \\
\Xi_{23} & =E^{T}\left(h_{21} Y_{12}^{T}-Y_{13}^{T}+Y_{23}\right) E, \\
\Xi_{24} & =E^{T}\left(h_{21} X_{12}-X_{13}+X_{23}^{T}+h_{21} Y_{12}-Y_{13}+Y_{23}^{T}\right) E, \\
\Xi_{25} & =h_{2} B^{T} R_{1}, \Xi_{26}=h_{21} B^{T} R_{2}, \\
\Xi_{33} & =-Q_{1}+E^{T}\left(h_{21} Y_{11}+Y_{13}+Y_{13}^{T}\right) E, \\
\Xi_{44} & =-Q_{2}+E^{T}\left(h_{21} Y_{22}-Y_{23}-Y_{23}^{T}+h_{21} X_{22}-X_{23}-X_{23}^{T}\right) E, \\
\Xi_{55} & -h_{2} R_{1}, \Xi_{66}=-h_{21} R_{2} .
\end{aligned}
$$

Proof: Consider the time-varying delay singular system (9), using the Lyapunov- Krasovskii functional candidate in the following form, we can write:

$$
\begin{aligned}
& V\left(x_{t}\right)=x^{T}(t) P E x(t)+\int_{t-h_{h}}^{t} x^{T}(s) Q_{1} x(s) d s+\int_{t-h_{2}}^{t} x^{T}(s) Q_{2} x(s) d s \\
& +\int_{t-h(t)}^{t} x^{T}(s) Q_{3} x(s) d s+\int_{-h_{2}}^{0} \int_{t+\theta}^{t} \dot{x}^{T}(s) E^{T} R_{1} E \dot{x}(s) d s d \theta \\
& +\int_{-h_{2}}^{-h_{t}} \int_{t+\theta}^{t} \dot{x}^{T}(s) E^{T} R_{2} E \dot{x}(s) d s d \theta
\end{aligned}
$$

Calculating the derivative of (11) with respect to $t>0$ along the trajectories of (9) leads to

$$
\begin{aligned}
& \dot{V}\left(x_{t}\right)=x^{T}(t)\left(P A+A^{T} P\right) x(t)+x^{T}(t) P B x(t-h(t)) \\
& +x^{T}(t-h(t)) B^{T} P x(t)+x^{T}(t)\left(Q_{1}+Q_{2}+Q_{3}\right) x(t) \\
& -x^{T}\left(t-h_{1}\right) Q_{1} x\left(t-h_{1}\right)-x^{T}\left(t-h_{2}\right) Q_{2} x\left(t-h_{2}\right) \\
& -x^{T}(t-h(t))(1-\dot{h}(t)) Q_{3} x(t-h(t))+\dot{x}^{T}(t) h_{2} E^{T} R_{1} E \dot{x}(t) \\
& +\dot{x}^{T}(t) h_{21} E^{T} R_{2} E \dot{x}(t)-\int_{t-h_{2}}^{t} \dot{x}^{T}(s) E^{T} R_{1} E \dot{x}(s) d s \\
& -\int_{t-h_{2}}^{t-h_{1}} \dot{x}^{T}(s) E^{T} R_{2} E \dot{x}(s) d s \\
& \leq x^{T}(t)\left(P A+A^{T} P+Q_{1}\right. \\
& \left.+Q_{2}+Q_{3}\right) x(t)+x^{T}(t) P B x(t-h(t)) \\
& +x^{T}(t-h(t)) B^{T} P x(t)-x^{T}\left(t-h_{1}\right) Q_{1} x\left(t-h_{1}\right) \\
& -x^{T}\left(t-h_{2}\right) Q_{2} x\left(t-h_{2}\right)- \\
& \quad{ }^{T}(t-h(t))\left(1-h_{d}\right) Q_{3} x(t-h(t)) \\
& \quad x \\
& +\dot{x}^{T}(t) h_{2} E^{T} R R_{1} E \dot{x}(t)+\dot{x}^{T}(t) h_{21} E^{T} R_{2} E \dot{x}(t) \\
& -\int_{t-h_{2}}^{t} \dot{x}^{T}(s) E^{T} R_{1} E \dot{x}(s) d s \\
& -\int_{t-h_{2}}^{t-h_{1}} \dot{x}^{T}(s) E^{T} R_{2} E \dot{x}(s) d s \\
& =x^{T}(t)\left(P A+A^{T} P+Q_{1}+Q_{2}+Q_{3}\right) x(t) \\
& +x^{T}(t) P B x(t-h(t)) \\
& +x^{T}(t-h(t)) B^{T} P x(t) \\
& \quad T \\
& -{ }^{T}\left(t-h_{1}\right) Q_{1} x\left(t-h_{1}\right) \\
& \quad x \\
& -x^{T}\left(t-h_{2}\right) Q_{2} x\left(t-h_{2}\right) \\
& -x^{T}(t-h(t))\left(1-h_{d}\right) Q_{3} x(t-h(t)) \\
& +\dot{x}^{T}(t) E^{T}\left(h_{2} R_{1}+h_{21} R_{2}\right) E \dot{x}(t) \\
& -\int_{t-h_{2}}^{t} \dot{x}^{T}(s) E^{T}\left(R_{1}-X_{33}\right) E \dot{x}(s) d s \\
& -\int_{t-h_{2}}^{t-h_{2}} \dot{x}^{T}(s) E^{T}\left(R_{2}-Y_{33}\right) E \dot{x}(s) d s \\
& -\int_{t-h_{2}}^{t-h_{2}} \dot{x}^{T}(s) E^{T} X_{33} E \dot{x}(s) d s \\
& E^{T} Y_{33} E \dot{x}(s) d s \\
&
\end{aligned}
$$

Noting the fact 
$-\int_{t-h_{2}}^{t} \dot{x}^{T}(s) E^{T} X_{33} E \dot{x}(s) d s$

$=-\int_{t-h_{2}}^{t-h(t)} \dot{x}^{T}(s) E^{T} X_{33} E \dot{x}(s) d s-\int_{t-h(t)}^{t} \dot{x}^{T}(s) E^{T} X_{33} E \dot{x}(s) d s$,

and

$$
\begin{aligned}
& -\int_{t-h_{2}}^{t-h_{1}} \dot{x}^{T}(s) E^{T} Y_{33} E \dot{x}(s) d s \\
& =-\int_{t-h_{2}}^{t-(t)} \dot{x}^{T}(s) E^{T} Y_{33} E \dot{x}(s) d s-\int_{t-h(t)}^{t-h_{1}} \dot{x}^{T}(s) E^{T} Y_{33} E \dot{x}(s) d s,
\end{aligned}
$$

Using the Leibniz-Newton formula $x(t)-x(t-h)=\int_{t-h}^{t} \dot{x}(s) d s$, and Lemma 2, we obtain:

$$
\begin{aligned}
& -\int_{t-h(t)}^{t} \dot{x}^{T}(s) X_{33} \dot{x}(s) d s \\
& \leq \int_{t-h(t)}^{t}\left[x^{T}(t) \quad x^{T}(t-h(t)) \quad \dot{x}^{T}(s)\right] \times \\
& {\left[\begin{array}{ccc}
X_{11} & X_{12} & X_{13} \\
X_{12}^{T} & X_{22} & X_{23} \\
X_{13}^{T} & X_{23}^{T} & 0
\end{array}\right]\left[\begin{array}{c}
x(t) \\
x(t-h(t)) \\
\dot{x}(s)
\end{array}\right] d s} \\
& \leq x^{T}(t) h_{2} X_{11} x(t)+x^{T}(t) h_{2} X_{12} x(t-h(t)) \\
& +x^{T}(t) X_{13} \int_{t-h(t)}^{t} \dot{x}(s) d s \\
& +x^{T}(t-h(t)) h X_{12}^{T} x(t) \\
& +x^{T}(t-h(t)) h_{2} X_{22} x(t-h(t)) \\
& +x^{T}(t-h(t)) X_{23} \int_{t-h(t)}^{t} \dot{x}(s) d s \\
& +\int_{t-h(t)}^{t} \dot{x}^{T}(s) d s X_{13}^{T} x(t)+\int_{t-h(t)}^{t} \dot{x}^{T}(s) d s X_{23}^{T} x(t-h(t)) \\
& =x^{T}(t)\left(h_{2} X_{11}+X_{13}+X_{13}^{T}\right) x(t) \\
& +x^{T}(t)\left(h_{2} X_{12}-X_{13}+X_{23}^{T}\right) x(t-h(t)) \\
& +x^{T}(t-h(t))\left(h_{2} X_{12}^{T}-X_{13}^{T}+X_{23}\right) x(t) \\
& +x^{T}(t-h(t))\left(h_{2} X_{22}-X_{23}-X_{23}^{T}\right) x(t-h(t))
\end{aligned}
$$

Similarly, we have

$$
\begin{aligned}
& -\int_{t-h_{2}}^{t-h(t)} \dot{x}^{T}(s) X_{33} \dot{x}(s) d s \\
& \leq x^{T}(t-h(t))\left(h_{21} X_{11}+X_{13}+X_{13}^{T}\right) x(t-h(t)) \\
& +x^{T}(t-h(t))\left(h_{21} X_{12}-X_{13}+X_{23}^{T}\right) x\left(t-h_{2}\right) \\
& +x^{T}\left(t-h_{2}\right)\left(h_{21} X_{12}^{T}-X_{13}^{T}+X_{23}\right) x(t-h(t)) \\
& +x^{T}\left(t-h_{2}\right)\left(h_{21} X_{22}-X_{23} X_{23}^{T}\right) x\left(t-h_{2}\right)
\end{aligned}
$$

$-\int_{t-h(t)}^{t-h_{h}} \dot{x}^{T}(s) Y_{33} \dot{x}(s) d s$

$$
\begin{aligned}
& \leq x^{T}\left(t-h_{1}\right)\left[h_{21} Y_{11}+Y_{13}^{T}+Y_{13}\right] x\left(t-h_{1}\right) \\
& +x^{T}\left(t-h_{1}\right)\left[h_{21} Y_{12}-Y_{13}+Y_{23}^{T}\right] x(t-h(t)) \\
& +x^{T}(t-h(t))\left[h_{21} Y_{12}^{T}-Y_{13}^{T}+Y_{23}\right] x\left(t-h_{1}\right) \\
& +x^{T}(t-h(t))\left[h_{21} Y_{22}-Y_{23}-Y_{23}^{T}\right] x(t-h(t))
\end{aligned}
$$

and

$$
\begin{aligned}
& -\int_{t-h_{2}}^{t-h(t)} \dot{x}^{T}(s) Y_{33} \dot{x}(s) d s \\
& \leq x^{T}(t-h(t))\left[h_{21} Y_{11}+Y_{13}^{T}+Y_{13}\right] x(t-h(t)) \\
& +x^{T}(t-h(t))\left[h_{21} Y_{12}-Y_{13}+Y_{23}^{T}\right] x\left(t-h_{2}\right) \\
& +x^{T}\left(t-h_{2}\right)\left[h_{21} Y_{12}^{T}-Y_{13}^{T}+Y_{23}\right] x(t-h(t)) \\
& +x^{T}\left(t-h_{2}\right)\left[h_{21} Y_{22}-Y_{23}-Y_{23}^{T}\right] x\left(t-h_{2}\right)
\end{aligned}
$$

With the operator for the term $\dot{x}^{T}(t) E^{T}\left(h_{2} R_{1}+h_{21} R_{2}\right) E \dot{x}(t)$ as follows:

$$
\begin{aligned}
& \dot{x}^{T}(t) E^{T}\left(h_{2} R_{1}+h_{21} R_{2}\right) E \dot{x}(t) \\
& =[x(t)+B x(t-h(t))]^{T}\left(h_{2} R_{1}+h_{21} R_{2}\right)[x(t)+B x(t-h(t))] \\
& =x^{T}(t) h A^{T}\left(h_{2} R_{1}+h_{21} R_{2}\right) A x(t) \\
& +x^{T}(t) h A^{T}\left(h_{2} R_{1}+h_{21} R_{2}\right) B x(t-h(t)) \\
& +x^{T}(t-h(t)) h^{T}\left(h_{2} R_{1}+h_{21} R_{2}\right) A x(t) \\
& +x^{T}(t-h(t)) h B^{T}\left(h_{2} R_{1}+h_{21} R_{2}\right) A x(t-h(t))
\end{aligned}
$$

To obtain an easily solvable LMI, we introduce the matrix $Z \in R^{n \times(n-r)}$ satisfying $E^{T} Z=0$ and rank $Z=n-r \quad[18,19$, 22].Then, we have

$$
0=2 \dot{x}^{T}(t) E^{T} Z S^{T} x(t)
$$

Substituting the above equations (13)-(20) into (12) yields

$$
\begin{aligned}
& \dot{V}\left(x_{t}\right) \leq \xi^{T}(t) \Omega \xi(t)-\int_{t-h_{2}}^{t} \dot{x}^{T}(s) E^{T}\left(R_{1}-X_{33}\right) E \dot{x}(s) d s \\
& -\int_{t-h_{2}}^{t-h_{1}} \dot{x}^{T}(s) E^{T}\left(R_{2}-Y_{33}\right) E \dot{x}(s) d s
\end{aligned}
$$

where $\quad \xi^{T}(t)=\left[\begin{array}{llll}x^{T}(t) & x^{T}(t-h(t)) & x^{T}\left(t-h_{1}\right) & x^{T}\left(t-h_{2}\right)\end{array}\right]$ and

$$
\Omega=\left[\begin{array}{cccc}
\Omega_{11} & \Omega_{12} & 0 & 0 \\
\Omega_{12}^{T} & \Omega_{22} & \Omega_{23} & \Omega_{24} \\
0 & \Omega_{23}^{T} & \Omega_{33} & 0 \\
0 & \Omega_{24}^{T} & 0 & \Omega_{44}
\end{array}\right],
$$

where

$$
\begin{aligned}
& \Omega_{11}=A^{T} P+P A+A^{T} Z S^{T}+S Z^{T} A+Q_{1}+Q_{2}+Q_{3} \\
& +E^{T}\left(h_{2} X_{11}+X_{13}+X_{13}^{T}\right) E+A^{T}\left(h_{2} R_{1}+h_{21} R_{2}\right) A, \\
& \Omega_{12}=P B+S Z^{T} B+E^{T}\left(h_{2} X_{12}-X_{13}+X_{23}^{T}\right) E \\
& +A^{T}\left(h_{2} R_{1}+h_{21} R_{2}\right) B, \\
& \Omega_{22}=-\left(1-h_{d}\right) Q_{3}+E^{T}\left(h_{21} X_{11}+X_{13}+X_{13}^{T}+h_{2} X_{22}-X_{23}-X_{23}^{T}\right. \\
& \left.+h_{21} Y_{11}+Y_{13}+Y_{13}^{T}+h_{21} Y_{22}-Y_{23}-Y_{23}^{T}\right) E+B^{T}\left(h_{2} R_{1}+h_{21} R_{2}\right) B, \\
& \Omega_{23}=E^{T}\left(h_{21} Y_{12}^{T}-Y_{13}^{T}+Y_{23}\right) E \\
& \Omega_{24}=E^{T}\left(h_{21} X_{12}-X_{13}+X_{23}^{T}+h_{21} Y_{12}-Y_{13}+Y_{23}^{T}\right) E, \\
& \Omega_{33}=-Q_{1}+E^{T}\left(h_{21} Y_{11}+Y_{13}+Y_{13}^{T}\right) E, \\
& \Omega_{44}=-Q_{2}+E^{T}\left(h_{21} Y_{22}-Y_{23}-Y_{23}^{T}+h_{21} X_{22}-X_{23}-X_{23}^{T}\right) E .
\end{aligned}
$$

If matrix inequality (21) is feasible, the by the Schur 
complement of Lemma 2 we can get $\Omega<0$, $E^{T}\left(R_{1}-X_{33}\right) E \geq 0, E^{T}\left(R_{2}-Y_{33}\right) \geq 0$ for any $\xi(t) \neq 0$. Therefore, the interval time-varying delay system (9) is asymptotically stable if (10) is true. Thus, the proof is complete.

\section{Robust for Uncertain Singular Interval Time-varying Delay System}

In the section, extending Theorem 1 to uncertain singular system (1) with interval time-varying delays yields the following Theorem 2 .

Theorem 2: For three given positive scalars $h_{1}, h_{2}$, and $h_{d}$, the uncertain singular time-varying delay system (1) is asymptotically stable if there exist symmetry positive-definite matrices $P=P^{T}>0, Q_{i}=Q_{i}^{T}>0$, $R_{j}=R_{j}^{T}>0 \quad(i=1,2,3 ; j=1,2) \quad \varepsilon>0, \quad S \quad$ with appropriate dimensions, positive semi-definite matrices $X=\left[\begin{array}{lll}X_{11} & X_{12} & X_{13} \\ X_{12}^{T} & X_{22} & X_{23} \\ X_{13}^{T} & X_{23}^{T} & X_{33}\end{array}\right] \geq 0$

and

$Y=\left[\begin{array}{lll}Y_{11} & Y_{12} & Y_{13} \\ Y_{12}^{T} & Y_{22} & Y_{23} \\ Y_{13}^{T} & Y_{23}^{T} & Y_{33}\end{array}\right] \geq 0$ such that the following

LMIs hold:

$$
\bar{\Xi}=\left[\begin{array}{ccccccc}
\bar{\Xi}_{11} & \bar{\Xi}_{12} & 0 & 0 & \Xi_{15} & \Xi_{16} & P M \\
\Xi_{12}^{T} & \bar{\Xi}_{22} & \Xi_{23} & \Xi_{24} & \Xi_{25} & \Xi_{26} & 0 \\
0 & \Xi_{23}^{T} & \Xi_{33} & 0 & 0 & 0 & 0 \\
0 & \Xi_{24}^{T} & 0 & \Xi_{44} & 0 & 0 & 0 \\
\Xi_{15}^{T} & \Xi_{25}^{T} & 0 & 0 & \Xi_{55} & 0 & h_{2} R_{1} M \\
\Xi_{16}^{T} & \Xi_{26}^{T} & 0 & 0 & 0 & \Xi_{66} & h_{21} R_{2} M \\
\Xi_{66} & 0 & 0 & 0 & h_{2} M^{T} R_{1} & h_{21} M^{T} R_{2} & -\varepsilon I
\end{array}\right]<0,
$$

$$
\begin{aligned}
& E^{T}\left(R_{1}-X_{33}\right) E \geq 0, \\
& E^{T}\left(R_{2}-Y_{33}\right) E \geq 0,
\end{aligned}
$$

with the following constraint

$$
P^{T} E=E^{T} P \geq 0 \text {. }
$$

where

$$
\bar{\Xi}_{11}=\Xi_{11}+\varepsilon N_{a}^{T} N_{a}, \bar{\Xi}_{12}=\Xi_{12}+\varepsilon N_{a}^{T} N_{b}, \bar{\Xi}_{22}=\Xi_{22}+\varepsilon N_{b}^{T} N_{b} \text {, }
$$$$
\Xi_{i j},(i, j=1,2, \ldots, 6 ; i<j \leq 6) \text { are defined in (10). }
$$

It is, incidentally, worth noting that the singular uncertain time-varying delay system (1) is asymptotically stable, that is, the uncertain parts of the nominal system can be tolerated within allowable time delay $h_{1} \leq h(t) \leq h_{2}$.

Proof: Replacing $A$ and $B$ in (10) with $A+M F(t) N_{a}$ and $B+M F(t) N_{b}$, respectively, we apply Lemma 4 [2] for system (1) is equivalent to the following condition:

$$
\Xi+\Gamma_{d} F(t) \Gamma_{e}+\Gamma_{e}^{T} F(t) \Gamma_{d}^{T}<0,
$$

where $\quad \Gamma_{d}=\left[\begin{array}{llllll}P M & 0 & 0 & 0 & h_{2} R_{1} M & h_{21} R_{2} M\end{array}\right] \quad$ and $\Gamma_{e}=\left[\begin{array}{llllll}N_{a} & N_{b} & 0 & 0 & 0 & 0\end{array}\right]$.

By lemma 4 [2], a sufficient condition guaranteeing (10) for system (1) is that there exists a positive number $\varepsilon>0$ such that

$$
\Xi+\varepsilon^{-1} \Gamma_{d}^{T} \Gamma_{d}+\varepsilon \Gamma_{e}^{T} \Gamma_{e}<0 .
$$

Applying the Schur complement shows that (24) is equivalent to (22a). This completes the proof.

When $h_{1}=0$, Theorem 1 reduces to the following Corollary 1 .

Corollary 1: For a give scalar $h>0, h_{d}>0$, the time-delay singular system (9) is asymptotically stable if there exist symmetric positive-definite matrices $P=P^{T}>0$, $Q=Q^{T}>0, \quad R=R^{T}>0$, and matrix $S$ of appropriate dimensions and a positive semi-definite matrix

$X=\left[\begin{array}{ccc}X_{11} & X_{12} & X_{13} \\ X_{12}^{T} & X_{22} & X_{23} \\ X_{13}^{T} & X_{23}^{T} & X_{33}\end{array}\right] \geq 0$ such that the following LMIs hold:

$$
\begin{gathered}
P^{T} E=E^{T} P \geq 0, \\
\Psi=\left[\begin{array}{ccc}
\Psi_{11} & \Psi_{12} & \Psi_{13} \\
\Psi_{12}^{T} & \Psi_{22} & \Psi_{23} \\
\Psi_{13}^{T} & \Psi_{23}^{T} & \Psi_{33}
\end{array}\right]<0,
\end{gathered}
$$

and

$$
E^{T}\left(R-X_{33}\right) E \geq 0
$$

where $Z \in R^{n \times(n-r)}$ is any matrix satisfying $E^{T} Z=0$ and

$$
\begin{aligned}
& \Psi_{11}=A^{T} P+P A+P A+A^{T} Z S^{T}+S Z^{T} A+Q \\
& +E^{T}\left(h X_{11}+X_{13}+X_{13}^{T}\right) E, \\
& \Psi_{12}=P A_{1}+S Z^{T} B+E^{T}\left(h X_{12}-X_{13}+X_{23}^{T}\right) E, \\
& \Psi_{13}=h A^{T} R, \Psi_{22}=-\left(1-h_{d}\right) Q+E^{T}\left(h X_{22}-X_{23}-X_{23}^{T}\right) E, \\
& \Psi_{23}=h B^{T} R, \Psi_{33}=-h R .
\end{aligned}
$$

Based on that, a convex optimization problem is formulated to find the bound on the allowable delay time $h$ which maintains the delay-dependent stability of the time delay system (9). 
Proof: Consider the singular time-varying delay system (9), using the Lyapunov- Krasovskii functional candidate in the following form, we can rewrite as

$$
\begin{aligned}
& V\left(x_{t}\right)=x^{T}(t) P E x(t)+\int_{t-h(t)}^{t} x^{T}(s) Q x(s) d s \\
& +\int_{-h}^{0} \int_{t+\theta}^{t} \dot{x}^{T}(s) E^{T} R E \dot{x}(s) d s d \theta
\end{aligned}
$$

Similar to the above analysis, one can get that $\dot{V}\left(x_{t}\right)<0$ holds if $\Psi<0$. Thus, the proof is completed.

Now, extending Corollary 1 to time delay singular uncertain system (1) with time-varying structured uncertainties yields the following Corollary2.

Corollary 2: For given scalar $h>0, h_{d}>0$, the time-delay singular system (1) is asymptotically stable if there exist symmetric positive-definite matrices $P=P^{T}>0$, $Q=Q^{T}>0, \quad R=R^{T}>0, \varepsilon>0, \quad$ and matrix $S$ of appropriate dimensions and a positive semi-definite matrix $X=\left[\begin{array}{lll}X_{11} & X_{12} & X_{13} \\ X_{12}^{T} & X_{22} & X_{23} \\ X_{13}^{T} & X_{23}^{T} & X_{33}\end{array}\right] \geq 0$ such that the following LMIs hold:

$$
\begin{gathered}
P^{T} E=E^{T} P \geq 0, \\
\bar{\Psi}=\left[\begin{array}{cccc}
\Psi_{11}+\varepsilon N_{a}^{T} N_{a} & \Psi_{12}+\varepsilon N_{a}^{T} N_{b} & \Psi_{13} & P M \\
\Psi_{12}^{T}+\varepsilon N_{b}^{T} N_{a} & \Psi_{22}+\varepsilon N_{b}^{T} N_{b} & \Psi_{23} & 0 \\
\Psi_{13}^{T} & \Psi_{23}^{T} & \Psi_{33} & h R M \\
M^{T} P & 0 & h M^{T} R & -\varepsilon I
\end{array}\right]<0
\end{gathered}
$$

and

$$
E^{T}\left(R-X_{33}\right) E \geq 0,
$$

where $Z$ follows the same definition as that in Theorem 1, and $\Psi_{i j}(i, j=1,2,3 ; i<j \leq 3)$ are defined in (25).

Remark 1: It is interesting to note that $h_{1}, h_{2}$, appear linearly in (10a) and (22a). Thus a generalized eigenvalue problem as defined in [2] can be formulated to solve the minimum acceptable $1 / h_{2}$ (or $1 / h_{1}$ ) and therefore the maximum $h_{2}\left(\right.$ or $\left.h_{1}\right)$ to maintain robust stability as judged by these conditions.

In this way, our optimization problem becomes a standard generalized eigrnvalue problem, then which can be solved using GEVP technique. From this discussion, we have the following Remark 2.

Remark 2: Theorem 2 provides delay-dependent asymptotic stability criteria for the time-varying delay singular systems (1) in terms of solvability of LMIs [2]. Based on them, we can obtain the maximum allowable delay bound (MADB) $h_{1} \leq h(t) \leq h_{2}$, such that (1) is stable by solving the following convex optimization problem:

$$
\begin{cases}\text { Maximize } & h_{2} \\ \text { Subject to } & \text { Theorem } 2\end{cases}
$$

\begin{tabular}{|c|c|c|c|c|c|}
\hline \multirow[b]{2}{*}{$h_{1}$} & & \multicolumn{4}{|c|}{$h_{d}$} \\
\hline & & 0.1 & 0.3 & 0.5 & 0.7 \\
\hline \multirow{2}{*}{0.1} & {$[16]$} & 0.5348 & 0.5318 & 0.5305 & 0.5300 \\
\hline & Corollary1 & 1.0484 & 1.0271 & 1.0213 & 1.0202 \\
\hline \multirow{2}{*}{0.2} & [16] & 0.5517 & 0.5414 & 0.5411 & 0.5410 \\
\hline & Corollary1 & 1.0485 & 1.0285 & 1.0241 & 1.0231 \\
\hline \multirow{2}{*}{0.3} & [16] & 0.5519 & 0.5421 & 0.5420 & 0.5411 \\
\hline & Corollary1 & 1.0487 & 1.0300 & 1.0268 & 1.0259 \\
\hline \multirow{2}{*}{0.4} & [16] & 0.5750 & 0.5690 & 0.5688 & 0.5684 \\
\hline & Corollary1 & 1.0488 & 1.0318 & 1.0295 & 1.0286 \\
\hline \multirow{2}{*}{0.5} & [16] & 0.6186 & 0.6154 & 0.6151 & 0.6150 \\
\hline & Corollary1 & 1.0490 & 1.0337 & 1.0321 & 1.0312 \\
\hline \multirow{2}{*}{0.6} & {$[16]$} & 0.6776 & 0.6759 & 0.6758 & 0.6755 \\
\hline & Corollary1 & 1.0493 & 1.0358 & 1.0347 & 1.0338 \\
\hline \multirow{2}{*}{0.7} & {$[16]$} & 0.7464 & 0.7460 & 0.7459 & 0.7458 \\
\hline & Corollary1 & 1.0495 & 1.0381 & 1.0372 & 1.0364 \\
\hline \multirow{2}{*}{0.8} & [16] & 0.8252 & 0.8250 & 0.8248 & 0.8245 \\
\hline & Corollary1 & 1.0498 & 1.0404 & 1.0396 & 1.0387 \\
\hline \multirow{2}{*}{0.9} & [16] & 0.9122 & 0.9121 & 0.9120 & 0.9118 \\
\hline & Corollary1 & 1.0502 & 1.0431 & 1.0426 & 1.0417 \\
\hline
\end{tabular}

Inequality (28) is a convex optimization problem and can be obtained efficiently using the MATLAB LMI Toolbox.

To show usefulness of our result, let us consider the following numerical examples.

Table 1. MADBs $h_{2}$ with given $h_{1}$ for different $h_{d}$ in example 1

\section{Illustrative Examples}

Example 1: Consider the following time delay singular systems:

$$
E \dot{x}(t)=A x(t)+B x(t-h(t))
$$

where $E=\left[\begin{array}{ll}1 & 0 \\ 0 & 0\end{array}\right], A=\left[\begin{array}{cc}0.5 & 0 \\ 0 & -1\end{array}\right], B=\left[\begin{array}{cc}-1.1 & 1 \\ 0 & 0.5\end{array}\right]$.

Find the delay-range $0 \leq h_{1} \leq h(t) \leq h_{2}$ to guarantee the above system (29) to be asymptotically stable.

Solution: Choosing $Z=\left[\begin{array}{ll}0 & 0 \\ 1 & 0\end{array}\right]^{\text {and taking the parameters }}$ $h_{1}=0.1$ and $h_{d}=0$ we get the Theorem 1 remains feasible 
for any delay time

$\left[h_{1}, h_{2}\right] \in[0.1,1.0641]$. Comparison with

other results is illustrated in Table 1. From Table 1, one can see that our results for this example give larger upper bounds of time-delay than the ones in [16]. By setting $h_{1}=0$ and different $h_{d}$ the upper bounds on the time delay from Corollary 1 are shown in Table 2, in which "." means that the results are not applicable to the corresponding cases. For comparison, the Table 2 also lists the upper bounds obtained from the criteria in $[1,6-8,17,19,20,22,23,26,28$, $29,31]$. It can be seen that our methods are less conservative. The simulation of the system (26) for $h=1.06$ is depicted in Fig.1 with the initial state $\left[\begin{array}{ll}-1 & 1\end{array}\right]^{T}$.

Table 2. MADBs $h_{2}$ for various $h_{d}$ in example 1

\begin{tabular}{|c|c|c|c|}
\hline \multirow[t]{2}{*}{ Methods } & \multicolumn{3}{|c|}{$h_{d}$} \\
\hline & 0 & 0.3 & 0.75 \\
\hline$[1,28]$ & - & - & - \\
\hline [29] & 0.5567 & - & - \\
\hline [8] & 0.8707 & - & - \\
\hline [6] & 0.9091 & - & - \\
\hline [7] & 0.9689 & - & - \\
\hline [26] & 1.0423 & - & - \\
\hline $\begin{array}{c}{[17,19,22,23,} \\
31]\end{array}$ & 1.0660 & - & - \\
\hline$[20]$ & 1.0660 & 1.0130 & 0.6496 \\
\hline Corollary 1 & 1.0660 & 1.0263 & 1.0164 \\
\hline
\end{tabular}

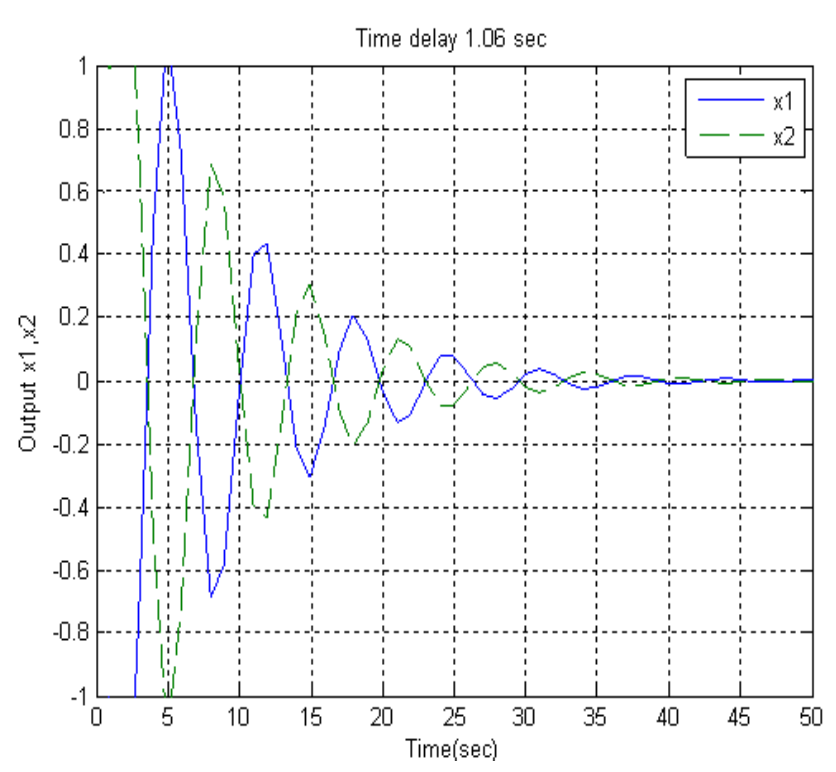

Figure 1. The simulation of the example 1 for $h=1.06 \mathrm{sec}$

Example 2: For convenience of comparison, consider the following time-delay system $[4,6,7,21]$.

$$
E \dot{x}(t)=A x(t)+B x(t-h(t))
$$

where $E=\left[\begin{array}{cc}-1 & 1 \\ 1 & -1\end{array}\right], A=\left[\begin{array}{cc}-1 & 0 \\ 0 & -1\end{array}\right], B=\left[\begin{array}{cc}1 & -1 \\ -1 & 1\end{array}\right]$.

Solution: In this example, we choose $Z=\left[\begin{array}{ll}0 & 1\end{array}\right]^{T}$. For $h_{1}=0$ and $h_{d}=0$ the system (30), we are able to find a feasible solution for the set of LMIs for any $\left[h_{1}, h_{2}\right] \in[0,1.1547]$. This means that the maximum allowable delay bound (MADB) under which the system is uniformly asymptotically stable is 1.1547 . Table 3 lists the maximum allowable delay bound (MADB) as judged by the criteria in $[4,6,7,21]$. We can see from this table that there is still room for reducing the conservativeness by comparing with the numerical solution, but we know that with fewer matrix variables the stability results obtained in Corollary 1 is less conservative than the one in $[4,6,7]$ and the same as [21]. Using these data, a simulation program has been written in Matlab. As Fig. 2 shows, the simulation of the above system (30) for $h_{2}=1.1547$ with the initial state $\left[\begin{array}{ll}-1 & 1\end{array}\right]^{T}$.

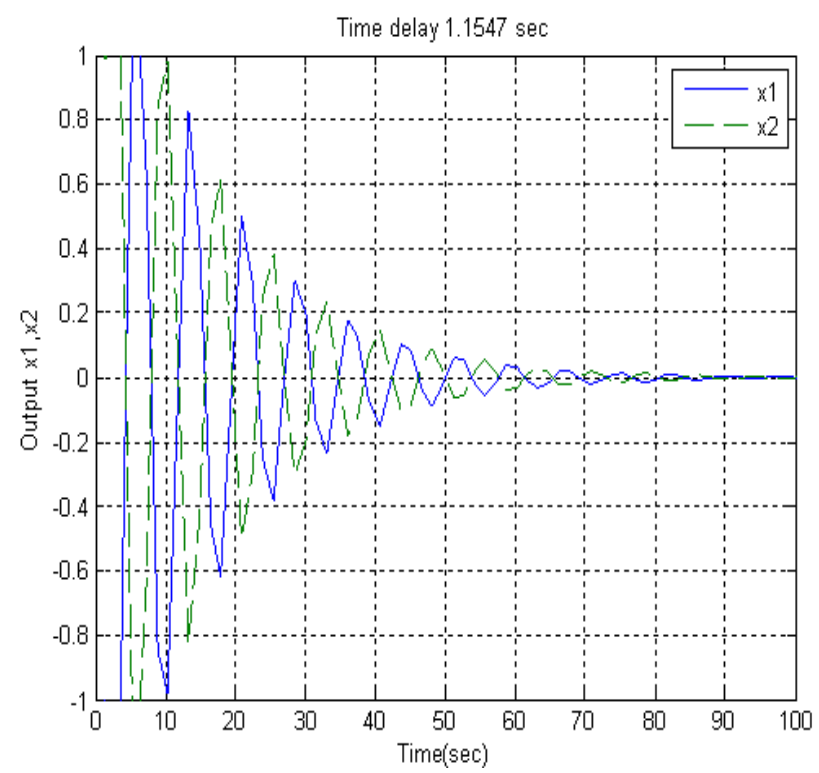

Figure 2. The simulation of the example 2 for $h=1.1547 \mathrm{sec}$

Table 3. Comparison of MADBs $h_{2}$ in example 2 for different methods and number of variables

\begin{tabular}{ccc}
\hline Methods & $h_{2}$ & $\begin{array}{c}\text { Number of } \\
\text { variables }\end{array}$ \\
\hline$[6]$ & $<1$ & 4 \\
{$[7]$} & 1.150 & 9 \\
{$[4]$} & 1.1547 & 7 \\
{$[21]$} & 1.1547 & 5 \\
Corollary 1 & 1.1547 & 5 \\
\hline
\end{tabular}

Example 3: Consider system (1) with respect to uncertainty (3) as follows: 


$$
E \dot{x}(t)=(A+\Delta A(t)) x(t)+(B+\Delta B(t)) x(t-h(t)),
$$

where

$$
\begin{aligned}
& E=\left[\begin{array}{cc}
1.0 & 0 \\
0 & 0
\end{array}\right], A=\left[\begin{array}{cc}
0.4 & 0.2 \\
0 & -1.0
\end{array}\right], B=\left[\begin{array}{cc}
-1 & 1.0 \\
0 & 0.3
\end{array}\right], \\
& M=\left[\begin{array}{cc}
0.1 & 0 \\
0 & 0.1
\end{array}\right], N_{a}=\left[\begin{array}{cc}
-0.05 & 0 \\
0 & 0.05
\end{array}\right], N_{b}=\left[\begin{array}{cc}
0.05 & 0 \\
0 & -0.05
\end{array}\right] .
\end{aligned}
$$

Solution: Choosing $Z=\left[\begin{array}{ll}0 & 0 \\ 1 & 0\end{array}\right]$ and taking the various parameters $h_{d}$, the computed maximum upper bounds of $h_{2}$, which guarantee the system (31) is regular, impulse free and robustly asymptotically stable for given bounds of $h_{1}$, are listed in Table 4 . Table 4 indicates that the time delay $h_{2}$ decreases when the $h_{d}$ increases. We claim that the sharpness of the upper bound delay time $h_{2}$ is negatively correlated with $h_{d}$. For $h_{1}=0$ and $h_{d}=0$ the system (31), we are able to find a feasible solution for the set of LMIs for any $\left[h_{1}, h_{2}\right] \in[0,1.1782]$. This means that the maximum allowable delay bound (MADB) under which the system is uniformly asymptotically stable is 1.1782 . Fig. 3 shows, the simulation of the above system (31) for $h_{2}=1.17$ with the initial state $\left[\begin{array}{ll}-1 & 1\end{array}\right]^{T}$.

Table 4. MADBs $h_{2}$ with given $h_{1}$ for varying $h_{d}$ in example 3 (Theorem 2)

\begin{tabular}{cccc}
\hline$h_{1}$ & \multicolumn{3}{c}{$h_{d}$} \\
\cline { 2 - 4 } & 0 & 0.3 & 0.75 \\
\hline 0.3 & 1.1816 & 1.1395 & 1.1255 \\
0.6 & 1.1816 & 1.1435 & 1.1342 \\
\hline
\end{tabular}

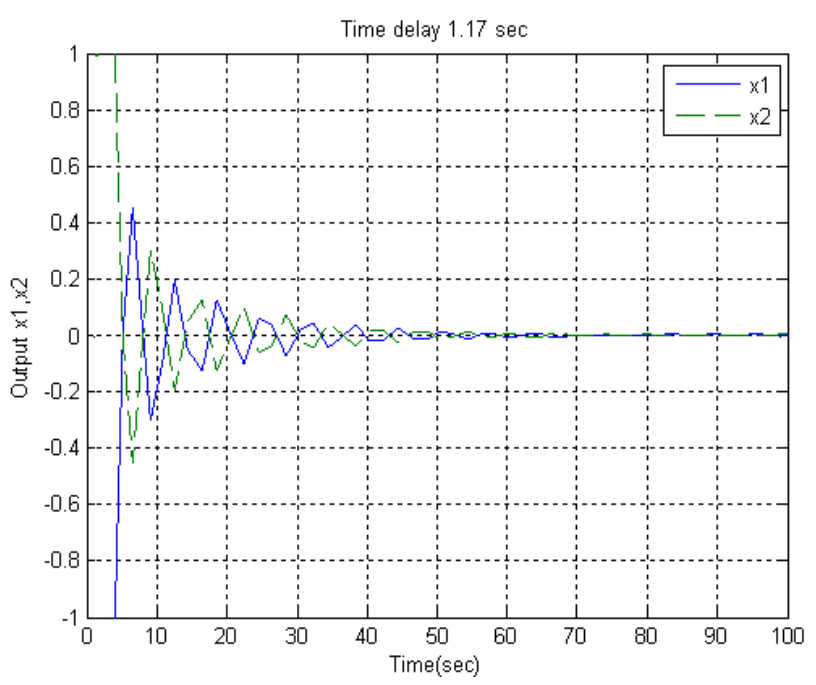

Figure 3. The simulation of the example 3 for $h=1.17 \mathrm{sec}$

\section{Conclusion}

In this paper, the improved delay-range-dependent robust stability criterion for uncertain singular time-delay systems with time-varying interval delays has been investigated. By defining a novel Lyapunov function, a delay-rangedependent stability criterion is established in terms of LMIs, which guarantees the nominal singular time-delay systems to be regular, impulse free and asymptotically stable. Less conservative result is obtained by considering some useful terms when estimating the upper bound of the derivative of Lyapunov functional and introducing the additional terms into the proposed Lyapunov function which includes the information of the range. The robust stability problem is also investigated and the obtained results are expressed in terms of strict LMIs, which can be easily solved by using a convex optimization algorithm. By comparing our results with others through numerical examples, it has been shown that the derived criterion is less conservative than those in the literature.

\section{REFERENCES}

[1] E. K. Boukas and Z. K. Liu, Delay-dependent stability analysis of singular linear continuous-time system, IEE Proceeding of Control Theory Application, Vol. 150, No.4, 325- 330, 2003.

[2] S L. Boyd, E. L. Ghaoui, E. Feron and V. Balakrishnan, Linear Matrix Inequalities in System and Control Theory, PA, Philadelphia: SIAM, 1994.

[3] L. Dai, Singular Control Systems, Berlin, Germany: Springer-Verlag, 1989.

[4] Y. Ding, S. W. Mei and X. Wang, Novel stability criteria of nonlinear uncertain systems with time- varying delay, Nonlinear Analysis: Real World Applications, Vol. 12, No.2,pp. 1152-1162, 2011.

[5] J. Feng, S. Zhu and Z. Cheng, Guaranteed cost control of linear uncertain singular time-delay systems, in Proceedings 41st IEEE Conference Decision and Control, 1802-1807, 2002.

[6] E. Fridman, Stability of linear descriptor systems with delay: A Lyapunov-based approach, Journal of Mathematical Analysis and Application, Vol. 273, No.1, 24 -44, 2002.

[7] E. Fridman and U. Shaked, $H_{\infty}$ control of linear state-delay descriptor systems: An LMI approach, Linear Algebra and Its Applications, Vol. 351, No.1, 271-302, 2002.

[8] H. Gao, S. Zhu, Z. Chen and B. Xu, Delay-dependent state feedback guaranteed cost control uncertain singular time-delay systems, in Proceeding of IEEE Conference on Decision and Control, and European Control Conference,4354-4359, 2003.

[9] K. Gu, S. Niculescu, Further remarks on additional dynamics in various model transformation of linear delay systems, IEEE Transactions on Automatic Control, Vol. 46, 497-500, 2001.

[10] J. K. Hale, Functional Differential Equations, New York: Springer-Verlag; 1997.

[11] Y. He, M. Wu, J. H. She and G. P. Liu, Parameter -dependent 
Lyapunov functional for stability of time- delay systems with polytopictype uncertainties, IEEE Transactions on Automatic Control, Vol. 49, No.3, 828 -832, 2004.

[12] X. Ji, H. Su and J. Chu, Delay-dependent robust stability of uncertain discrete singular time-delay systems, in Proceedings of the American Control Conference, 3843-3848, 2006.

[13] Z. Jiang, W. Gui, Y. Xie and H. Wu, Delay-dependent stabilization of singular linear continuous-time systems with time-varying state and input delays, in Proceedings IEEE Conference Control and Automation, Guangzhou, China, $1862-1867,2007$.

[14] V. B. Kolmanovskii and V. R. Nosov, Stability of Functional Differential Equations, New York: Academic, 1986.

[15] J. X. Lin, X. L, Zhao, and S. M. Fei, New delay-range-dependent exponential estimates for singular systems with time-varying delay, International Journal of Control, Automation and Systems, Vol. 9, No. 2, pp 218-227, 2011.

[16] F. L. Lewis, A survey of linear singular systems, Circuits Systems Signal Processing, Vol. 5, 3-36, 1986.

[17] P. L. Liu, Further results on the exponential stability criteria for time delay singular systems with delay- dependence, International Journal of Innovative Computing, Information and Control, Vol. 8, No. 6, 4015-4024, 2012.

[18] P. L. Liu, Further results on the stability analysis of singular systems with time-varying delay: A delay decomposition approach, International Journal of Analysis, ID 721407, 1-11, 2013.

[19] H. Su, X. Ji and J. Chu, Delay-dependent robust control for uncertain singular time-delay systems, Asian Journal of Control, Vol. 8, No.2, 1-10, 2006.

[20] H. Wang, A. Xue, R. Lu and J. Wang, Delay- dependent robust stability and stabilization for uncertain singular system with time-varying delay, in Proceedings American Control Conference, 1327-1331, 2008.

[21] C. H. Wang, New delay-dependent stability criteria for descriptor systems with interval delay, Asian Journal of Control, Vol. 14, No.1, 197-206, 2012.

[22] Z. Wu and W. Zhou, Delay-dependent robust $H_{\infty}$ control for uncertain singular time-delay systems, IEE Proceeding-Control Theory Application, Vol. 1, No.5, 1234-1241, 2007.

[23] Z. Wu and W. Zhou, Delay-dependent robust stabilization for uncertain singular systems with state delay, Acta Automatica Sinica, Vol. 33, No.3, 714-718, 2007.

[24] S. Xu, P. Van Dooren, R. Stefan and J. Lam, Robust stability and stabilization for singular systems with state delay and parameter uncertainty, IEEE Transactions on Automatic Control, Vol. 47, No.7, 1122 -1128, 2002.

[25] S. Xu, J. Lam and L. Zhang, Robust D-Stability analysis for uncertain discrete singular systems with state delay, IEEE Transactions on Circuits and Systems I-Regular, Vol. 49, No.4, 551-5552, 2002.

[26] F. Yang and Q. Zhang, Delay-dependent $H_{\infty}$ control for linear descriptor systems with delay in state, Journal of Control Theory and Application, Vol. 3, No.1, 76-84, 2005.
[27] D. Yue and Q. L. Han, A delay-dependent stability criterion of neutral systems and its application to a partial element equivalent circuit model, IEEE Transactions on Circuits and Systems-II, Express Briefs, Vol. 51, No.12, 685-689, 2004.

[28] R. Zhong and Z. Yang, Robust stability analysis of singular linear system with delay and parameter uncertainty, Journal of Control Theory and Application, Vol. 3, No.2, 195-199, 2005.

[29] R. Zhong and Z. Yang, Delay-dependent robust control of descriptor systems with time delay, Asian Journal of Control, Vol. 8, No.1, 36-44, 2006.

[30] S. Zhou and J. Lam, Robust stabilization of delayed singular systems with linear fractional parametric uncertainties, Circuits and Systems, Signal Processing, Vol. 22,578-588, 2003.

[31] S. Zhu, C. Zhang, Z. Chen and J. Feng, Delay- dependent robust stability criteria for two classes of uncertain singular time-delay systems, IEEE Transactions on Automatic Control, Vol. 52, No.5, 880-885, 2002. 